\title{
On the applicability of quantum-optical concepts in strong-coupling nanophotonics
}

\author{
C. Tserkezis,, , * A. I. Fernández-Domínguez, ${ }^{2}$ P. A. D. Gonçalves,${ }^{1,3,4}$ F. Todisco, ${ }^{1}$ J. D. Cox,${ }^{1,5}$ \\ K. Busch, ${ }^{6,7}$ N. Stenger, ${ }^{3,4}$ S. I. Bozhevolnyi, ${ }^{1,5}$ N. A. Mortensen, ${ }^{1,3,5}$, † and C. Wolff 1 , 团 \\ ${ }^{1}$ Center for Nano Optics, University of Southern Denmark, Campusvej 55, DK-5230 Odense M, Denmark \\ ${ }^{2}$ Departamento de Física Teórica de la Materia Condensada and Condensed Matter Physics Center (IFIMAC), \\ Universidad Autónoma de Madrid, E-28049 Madrid, Spain \\ ${ }^{3}$ Center for Nanostructured Graphene, Technical University of Denmark, DK-2800 Kongens Lyngby, Denmark \\ ${ }^{4}$ Department of Photonics Engineering, Technical University of Denmark, DK-2800 Kongens Lyngby, Denmark \\ ${ }^{5}$ Danish Institute for Advanced Study, University of Southern Denmark, Campusvej 55, DK-5230 Odense M, Denmark \\ ${ }^{6}$ Max-Born-Institut, Max-Born-Straße 2A, D-12489 Berlin, Germany \\ ${ }^{7}$ Humboldt-Universität zu Berlin, Institut für Physik, \\ AG Theoretische Optik \& Photonik, Newtonstraße 15, D-12489 Berlin, Germany
}

\begin{abstract}
Rooted in quantum optics and benefiting from its well-established foundations, strong coupling in nanophotonics has experienced increasing popularity in recent years. With nanophotonics being an experiment-driven field, the absence of appropriate theoretical methods to describe ground-breaking advances has often emerged as an important issue. To address this problem, the temptation to directly transfer and extend concepts already available from quantum optics is strong, even if a rigorous justification is not always available. In this Review we discuss situations where, in our view, this strategy has indeed overstepped its bounds. We focus on exciton-plasmon interactions, and particularly on the idea of calculating the number of excitons involved in the coupling. We analyse how, starting from an unfounded interpretation of the term $N / V$ that appears in theoretical descriptions at different levels of complexity, one might be tempted to make independent assumptions for what the number $N$ and the volume $V$ are, and attempt to calculate them separately. Such an approach can lead to different, often contradictory results, depending on the initial assumptions (e.g. through different treatments of $V$ as the - ambiguous in plasmonics - mode volume). We argue that the source of such contradictions is the question itself - How many excitons are coupled?, which disregards the true nature of the coupled components of the system, has no meaning and often not even any practical importance. If one is interested in validating the quantum nature of the system - which appears to be the motivation driving the pursuit of strong coupling with small $N$ - one could instead focus on quantities such as the photon emission rate or the second-order correlation function. While many of the issues discussed here may appear straightforward to specialists, our target audience is predominantly newcomers to the field, either students or scientists specialised in different disciplines. We have thus tried to minimise the occurrence of proofs and overly-technical details, and instead provide a qualitative discussion of analyses that should be avoided, hoping to facilitate further growth of this promising area.
\end{abstract}

\section{INTRODUCTION}

The use of analogies and transference of concepts from other scientific areas, particularly solid-state physics and quantum optics, has contributed greatly to the rapid progress nanophotonics has experienced in recent years. This is illustrated, for instance, by the development of photonic crystals [1], the quest for Anderson localisation of light [2], or plasmon-induced transparency [3]. A more recent example can be identified in the increasingly popular exploration of strong coupling in light-matter interactions at the nanoscale. Starting from the purely quantum Rabi problem for a two-level system in a dielectric microcavity [4], this area of light-matter interactions has gradually shifted towards systems exhibiting (semi)classical characteristics, with stronger (possibly

\footnotetext{
*Electronic address: ct@mci.sdu.dk

${ }^{\dagger}$ Electronic address: asger@mailaps.org

${ }^{\ddagger}$ Electronic address: cwo@mci.sdu.dk
}

collective) transition dipole moments and modes confined in ever smaller subwavelength cavities. More than merely an analogy, this shift of attention has likely occurred from the necessity to design experimental set-ups that test and utilise quantum-optical theories while providing a platform for the realisation of practical quantum technologies. The initial study of atoms placed between dielectric mirrors - the playground of cavity quantum electrodynamics (cQED) [5] - gave thus its place to quantum wells between Bragg reflectors [6], quantum dots in photonic crystals [7], and more recently to excitons in molecular aggregates or transition-metal dichalcogenides (TMDs) interacting with plasmonic nanoparticles (NPs) [8 10].

In the pursuit of stronger emitter-cavity interactions exhibiting wider avoided crossings between the initial bare states (Rabi splitting), which will potentially result in stronger quantum and nonlinear effects 11, 12, one constant guide has been the factor $N / V$ [13], where $N$ is the number of emitters, and $V$ a volume to be discussed, typically of the cavity or the associated optical mode. In a purely classical description of coupled harmonic oscillators, this term appears directly as a density of emit- 
ters (more generally, dipole moments) [13]. On the other hand, from the standpoint of cQED, an analysis based on the Tavis-Cummings Hamiltonian 14 shows that the collective coupling strength $g$ of $N$ two-level emitters in a low-loss, nonradiative, single-mode cavity of volume $V$ is $g \propto \sqrt{N / V}[15]$, where $V$ emerges as a result of the particle-in-a-box quantisation of the EM fields. The vast majority of experimental and theoretical efforts in nanophotonics has focused exactly on enhancing $g$, either by directly controlling the density of emitters [16], or by separately increasing the numerator or decreasing the denominator of $N / V$. In the latter approach, the number of emitters can be precisely controlled when atoms, quantum dots, or vacancies in solid-state platforms are involved. This, however, is not necessarily the case when dealing with the molecular $J$-aggregates that have recently been introduced in nanophotonics [17, 18], for which one can indeed question the meaning of $N$, as we discuss below. Current efforts are more devoted on minimising $V$ through the design of subwavelength cavities [19], aiming now not only to achieve as large a $g$ as possible, but do so with the smallest $N$, so as to potentially enter the quantum regime; this is where plasmonics comes into play. With these complementary recipes, extremely high coupling strengths have been achieved, with Rabi splittings on the order of hundreds of $\mathrm{meV}$ (for an overview of recent experimental results see for example 20]), even entering the ultrastrong coupling regime 21 23], where the rotating wave approximation, which neglects rapidly oscillating terms, no longer holds.

Despite these achievements, however, to quote Hugall et al. 24], the efforts of nanophotonics are "rarely taken seriously in the context of $c Q E D$." Apart from the persistent, ever-present issue of Ohmic loss [25], one possible reason for this could be that in nanophotonics, on some occasions and driven by the need to shed light on experimental findings, concepts from other fields have been straightforwardly adapted and applied outside their original context. Here we discuss some recent trends that in our view are concerning, focusing mainly on the attempt to estimate the number of excitons participating in the collective strong coupling phenomena [26 29]. We would like to stress that our goal is simply to call attention to such misconceptions and, hopefully, contribute thus to the development of the field on more solid ground. In doing so, we do not intend to undervalue any previous works - some of the potential errors that we discuss below might be found in some of our papers as well [30] - and our arguments do not invalidate any publication in its entirety; only small parts of the analysis presented in some works are criticised. Evidently, different topics touched upon here might appear obvious to readers with different backgrounds. This Review is therefore addressed mostly to newcomers and students, but if controversy emerges, it should act as an open invitation to fruitful discussions, which will only help nanophotonics further advance: cQED already benefited from such debates a few decades ago 31]. Naturally, there are several occasions where we agree that strong coupling was addressed in a careful and productive manner and could act as guidelines for future research, and some of these are mentioned in the last section.

\section{ANALYSIS OF STRONG COUPLING AND PRACTICES THAT CALL FOR ATTENTION}

\section{A. The origin of $\mathrm{N} / \mathrm{V}$ in the coupling}

In our experience, the origin of most controversies lies in the temptation to rely, in every kind of situation, on the readily accessible but not always applicable equation for the coupling strength [32 34] [119],

$$
g=\frac{1}{\hbar} \sqrt{N} \boldsymbol{\mu} \cdot \mathbf{E}=\mu \sqrt{\frac{\omega N}{2 \hbar \varepsilon \varepsilon_{0} V}} \propto \sqrt{\frac{N}{V}},
$$

where $\boldsymbol{\mu}$ is the dipole moment of each one of $N$ identical emitters, $\mathbf{E}$ is the homogeneous electric field they experience in a good photonic cavity of volume $V, \omega$ the angular frequency of the light, and $\varepsilon_{0}$ and $\varepsilon$ the vacuum and cavity permittivity, respectively. This is the result of a fully quantum description, where the emitters are described as two-level systems and the electromagnetic (EM) field is quantised into orthonormal modes of a resonator. On the right-hand side of equation (11) we have deliberately isolated the term $\sqrt{N / V}$, on which we focus here, because it does not necessarily require a fully quantum description to emerge. In order to discuss why it is not always interpreted correctly, it is useful to first briefly consider the different ways to obtain it, namely a classical, semiclassical, and quantum description of the problem, following the analysis previously outlined in [13]. It is also useful to note at this point that the square-root (rather than linear) dependence of the mode splitting on $N$ is strong evidence of the collective response of the ensemble of $N$ individual emitters, and offers thus an experimentally accessible tool for verifying the origin of any observed anticrossing.

With strong coupling in nanophotonics involving large aggregates of organic molecules or layered TMDs (where the dynamics of excitons is strongly influenced by longrange Coulomb interactions) combined with extended metallic films or small (but macroscopic) plasmonic NPs, a purely classical description can already capture most of the observed effects. In this case, one can start, as a first approximation, by considering a single electron of the emitter layer as a damped harmonic oscillator, driven by the external - homogeneous and harmonic EM field, with its dipole moment acquiring the form of a classical Lorentzian oscillator. In a macroscopic medium with $N$ dipoles per unit volume $V$, the polarisation density $\mathbf{P}$ is defined as the average dipole moment per unit volume [35], from which the resulting susceptibility and permittivity of the emitter layer depend on $N / V$. In the simple example of a dye layer coupled to the sur- 


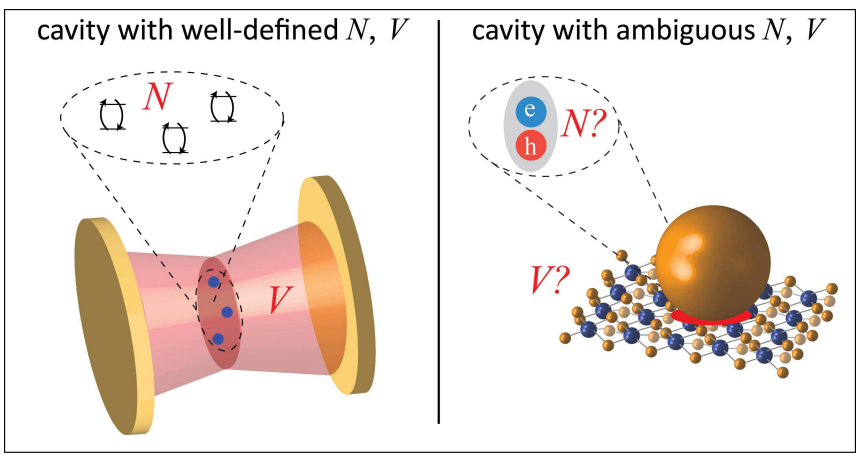

FIG. 1: Schematic of the physical problem under study. On the left-hand panel, a collection of $N$ individual two-level systems is placed between two mirrors forming a dielectric microcavity of volume $V$. Both $N$ and $V$ are well-defined quantities, and using the Tavis-Cummings picture makes sense. On the right-hand panel, excitons in a TMD interact with a plasmon mode of a metallic NP. Both $N$ and $V$ are ambiguous, and usage of the $N / V$ factor is questionable.

face plasmons of an underlying metal film, one can introduce the dye permittivity in the plasmon dispersion, leading to a quadratic equation with two branches separated by a band gap whose width is proportional to $\sqrt{N / V}$. A larger effective density of dipoles provides thus the straightforward means to obtain wider stop bands, i.e. mode splittings, as has been shown experimentally [36], and this density is the only relevant parameter.

In a semiclassical description, the EM field remains classical but the homogeneous emitter layer is replaced by a collection of two-level quantum emitters. Starting with the case of a single emitter, the Hamiltonian of the system, within the rotating-wave approximation and after the appropriate unitary transformations, is written as

$$
H=-\frac{\hbar \delta}{2}+\frac{\hbar \Omega_{\mathrm{R}}}{2}\left(\sigma_{+}+\sigma_{-}\right),
$$

where $\Omega_{\mathrm{R}}$ is the Rabi frequency, $\delta=\omega-\omega_{0}$ is the detuning between the frequency of the optical mode $\omega$ (say the surface plasmon, to relate with the classical description) and the frequency $\omega_{0}$ of a transition from the excited to the ground state of the emitter, and $\sigma_{ \pm}$are the standard Pauli matrices [37]. Making the extension to a collection of such emitters, and calculating the expectation value of the induced dipole moment, it can be shown (for details see [13]) that the polarisation density for $N$ emitters per unit volume is again related to the density $N / V$, with $\Omega_{\mathrm{R}}(=2 g) \propto \sqrt{N / V}$. When $N$ emitters oscillate in phase, the obtained Rabi splitting is proportional to the emitter density [120].

It is only in a fully quantum description, where the EM field is also quantised and both the emitter and the field are described by appropriate creation and annihilation operators, that the two factors, $N$ and $V$, can be rigorously defined separately. As implied by equation (11), $V$ is in this case directly related to the EM field and, in partic- ular, to its particle-in-a-box normalisation. In this case, the system is described by the Tavis-Cummings Hamiltonian, which is often solved for simplicity in the large$N$ limit, with only a small fraction of the total emitters being excited. This allows to map the many two-level (fermionic) emitters to a large bosonic super-oscillator, to obtain $\Omega_{\mathrm{R}}=2 g$ with $g$ given by equation (1). For $N=1$, the first equality in equation (1) is thus an accurate starting point, as it describes light-matter interactions within the dipole approximation [37, 38], although one could, and in some situations should, go one step backwards, e.g. by introducing the electron-photon interaction Hamiltonian in the appropriate gauge to ensure that higher-order interaction terms are included [39, 40]. Using the same equations for $N>1$ yet distinguishable emitters, however, requires that all dipole moments experience a uniform electric field of the cavity and (for the second equality) they are aligned. For atoms in extended microcavities excited by a laser field, a description in terms of two-level systems with the same dipole moment experiencing the same field is valid, and an analysis based on equation (11) can safely apply [41, 42]. In contrast, in plasmonics, where the cavities are i) open, ii) comparable in size with the emitters themselves, and iii) lossy, such an approach must be judiciously adopted, as recent experiments corroborate 43]. Below we discuss some of the ambiguities that may arise in defining $V$ and $N$, shown schematically in Figure 1, as they appear in recent literature.

\section{B. Mode volume: is it always the same?}

The mode volume enters equation (10) as the normalisation constant for the quantised field, denoting the volume of a hypothetical cavity that would provide the same maximum field per photon [4]. But this already requires that the field can be quantised in an unambiguous manner. Whether and how one can define a mode volume for an open, dispersive cavity with high Ohmic and radiative losses is still under debate. The simple relation based on energy density [45] — often suitable for dielectric cavities - no longer holds, and a first required action is to at least account for the dispersive character of the metal [46, 47]. In such an approach one usually normalises a volume integral of the energy density to its maximum value. But particular care must be taken here: such a normalisation might be an acceptable starting point to describe the plasmonic mode itself, but is not necessarily extendable to the emitter-cavity problem, as we discuss below. More rigorous definitions and derivations of mode volumes have been obtained in recent years in the context of quasinormal modes (QNMs) [48 50], obtained as the solutions $\tilde{\mathbf{f}}_{\mu}$ of the Helmholtz equation with open boundary conditions,

$$
\nabla \times \nabla \times \tilde{\mathrm{f}}_{\nu}(\mathrm{r})-\left(\frac{\tilde{\omega}_{\nu}}{c}\right)^{2} \varepsilon(\mathrm{r}, \omega) \tilde{\mathrm{f}}_{\nu}(\mathrm{r})=0,
$$


where $\tilde{\omega}_{\nu}$ is the complex resonance frequency of mode $\nu$, and $\varepsilon(\mathbf{r}, \omega)$ the position $(\mathbf{r})$ - and frequency-dependent permittivity of the cavity, a formalism which even allows to straightforwardly implement quantum corrections [51]. Once these solutions are normalised, one can expand any field in a set of QNMs, construct the Green's function, and in principle obtain the solution to any EM problem. But the exact normalisation is still under debate: it is for instance an open question whether it is preferable to base a QNM formulation on a Green's function expansion [52, 53] or a field expansion [48, 54, 55]. While we do not have a clear answer to this, we believe that QNMs, or possibly hybrid schemes with elements from both a quasi- and a pseudo-mode picture [56], even if not always practical for an immediate analysis [57, constitute a promising prospect. For more details about different approaches to QNMs we refer the reader to the Perspective by Kristensen and Hughes [58], the Review by Lalanne et al. [59], or the more recent Tutorial by Kristensen et al. [60].

In the context of emitter-cavity coupling, a frequent approach to obtain a first estimate for the volume that enters equation (11) is based on simple geometric arguments. For example, what is sometimes used as $V$ is the geometric volume of the plasmonic NP [61]. The reasoning behind this choice is based on the small dimensions of the NP (thus less radiative), and the fact that the energy is confined inside it. While this may offer a rough qualitative estimate for the plasmonic mode volume itself — within an order of magnitude as compared e.g. to QNM approaches — this approach is not particularly accurate for emitter-plasmon coupling. Here, the volume that matters is only that which is relevant to the emitter, and appears in calculations based on the local density of states (LDOS) 62, 63] through the appropriate Green's function 64. It is clearly mentioned for instance by Shahbazyan [65] that with such robust calculations one can only obtain a local mode volume. And since volume integrals always appear in the relevant expressions, it is crucial to distinguish between integrating over the entire space or over the entire cavity. In such situations, single NPs, or their assemblies, do not constitute the cavity, but resemble more a combination of the exciting source and the mirror at the cavity end. On the one hand, it is localised plasmon resonances that generate the enhanced near field where the emitters are placed, boosting thereby the LDOS (source part). On the other hand, these are also the origins of loss, through radiation and absorption, so that in this respect they are (bad) cavity mirrors. Eventually, what is really relevant is the electric field confined near or between NPs (and/or a possible substrate), where the emitters are located [66]. For rough estimates of orders of magnitude, a better though still loose - approximation would be thus to just consider the geometric volume of the cavity, e.g. the volume between two spheres in a plasmonic dimer [67].

Finally, another mode-volume calculation that occasionally appears in literature, is the one related to the
Purcell factor [68],

$$
P=\frac{3 Q}{4 \pi^{2} V}\left(\frac{\lambda}{n}\right)^{3}
$$

where $n=\sqrt{\varepsilon}$ is the refractive index and $Q$ the quality factor of the cavity. Since $P$ can be evaluated (typically with numerical calculations) from the shortening of the excitation lifetime, and $Q$ is given by the resonance linewidth, obtaining $V$ from equation (4) appears tempting, and a Letter by Koenderink 62 is often cited as the reference which justifies its use. However, as clearly stated in that Letter, this equation holds only as long as a) normal modes can be defined for the system, so that the LDOS is written as a sum of such, and b) this sum is dominated by a single mode. This is usually not the case in plasmonics, especially as far as the singlemode requirement is concerned, and the shortening of the lifetime is often different (larger) than the emission intensity enhancement due to the coupling to both radiative and nonradiative plasmonic modes. Nevertheless, equation (4) is still in use - although usually not as a means to obtain quantitative results [19] - even though the very occurrence of strong coupling and the suppression of the well-known fluorescence quenching [69] occurs exactly because the emitters also interact with a pseudomode formed by all higher-order nonradiative modes [64, 70, 71]. We could not help noticing that situations of using the equations in 62 out of context must be so frequent, that its author felt the need to clarify in a recent paper that "we use the term mode volume here not as an endorsement of the validity of this concept per se for plasmonics" [72].

To summarise this subsection, the main idea we try to convey is that the appearance of a $V$ in an equation does not necessarily always mean the same volume: different effects should require a different treatment. In this respect, the volume that enters normalisation of the plasmonic field, calculated either through the energy density or QNMs, is not the same as the one that is relevant to an emitter, which is related to the LDOS the emitter experiences at points outside the cavity. The concept of different mode volumes is well-established, for example in the context of plasmonic waveguides [73].

\section{Number of emitters: should one count excitons?}

The previous subsection discussed different ways to estimate a mode volume, and the ambiguities that emerge in the case of plasmonics. But even if it would eventually be calculated with a universally accepted method, the question that lies at the heart of this Review is whether that volume should in turn be used to evaluate the number of emitters coupled to a plasmonic cavity from the observed mode splitting. In 2016, Chikkaraddy et al. [19] studied a carefully designed cavity where individual molecular emitters, encapsulated in barrel-shaped 
molecules so as to prevent them from interacting with each other and align their dipole moments with the plasmonic near field, were placed in ultrasmall NP-on-mirror cavities. Statistical analysis of the data showed that, in some situations, the occurring anticrossing could be attributed to interaction of the cavity with just one emitter, thus reporting single-molecule strong coupling at room temperature. This result, together with the work published on the same day by Santhosh et al. 74], involving a single quantum dot in the middle of a plasmonic bowtie antenna, has set strong-coupling nanophotonics on a new basis. But in the wake of the enthusiasm that followed those papers, a number of other works seem to have initiated - albeit unintentionally - an undeclared competition for the strongest coupling with the smallest number of emitters (presumably with the intention to enter the quantum-optical regime), which is still growing today. Even though the conclusions of those seminal experiments were not based on equation (1), the $N / V$ factor has turned into the most easily accessible analysis tool in recent literature, either merely for qualitative discussions (where it can be legitimate) or even to obtain quantitative results $[23,28,30,34,75]$. In the latter case, $g$ is usually first estimated from the experimentally or numerically observed spectral anticrossing as $g=\Omega_{\mathrm{R}} / 2$ (assuming a lossless system). If the values of both $N$ and $V$ are well-defined and thus meaningful, the coupling strength obtained from their ratio is then compared to the observed $g$ to check the validity of equation (1) and verify that the strong-coupling regime has been reached. This control can of course also be done when the density $N / V$ is directly available. But when collective excitations are involved and $N$ is ill-defined, researchers have nevertheless been tempted to use $g$ and the $V$ obtained either from rough geometrical estimates [67, 76], or numerically as described in the previous subsection, to evaluate $N$. Whether such a calculation is meaningful, is the topic of this subsection.

Ever since excitons in $J$-aggregates of organic molecules were first proposed as systems with a higher dipole moment, which can thus facilitate strong coupling in plasmonics [8, 77], several attempts to extend the use of equation (11) to any strong-coupling set-up, regardless of the nature of the emitter, have been made. In particular, excitons in TMDs coupled to plasmonic NPs have emerged as an attractive architecture [26, 27, 29]; yet in the case of TMDs - whose complex excitonic resonances are described beyond a single-particle picture [78] - this approach has to be questioned: this is really more a problem of interpretation than of the calculation itself. It is of course always possible to calculate a value $N$ from a coupling constant $g$, a mode volume $V$ and a permittivity $\varepsilon$ (whatever the appropriate one might be for a plasmonic resonator on a substrate in air), but it is not clear what this - in general non-integer $-N$ might be counting. As we show further below, it certainly does not count the number of excitons that are coupled to the plasmonic resonator in the way isolated molecules do.
Our main argument against this practice is based on the apparent mix-up between a physical system (as described, e.g., by the Hamiltonian) and its states. Single molecules (or atoms) are clearly part of the system, represented by a pair of raising and lowering operators $\hat{f}^{\dagger}$ and $\hat{f}$ per molecule in the Hamiltonian. In contrast, an exciton is not part of the bare system, and the excitonic population does not appear directly or indirectly anywhere in the Hamiltonian. An exciton is an excitation of the system - hence the name. The excitonic population is an observable, associated with one of several quantum numbers that can be used to label the state of the system. When compared to a single quantum emitter like an atom or colour center, the exciton does not correspond to an emitter, but rather to a transition within an effective emitter. A schematic representation of the differences between atom-like and excitonic emitters is shown in Figure 2 In the upper left-hand panel, the energy ladder of a single two-level system is shown. For $N$ emitters one has to simply imagine $N$ identical such ladders. Instead, in TMDs there exists an extended distribution of excitons - and the excitonic gas is bosonic (or at least nearly so) 79 81. While the classification of excitons in TMDs is not perfectly clear, the fact that their wavefunctions have the form of Wannier-Mott excitons [82, 83], implies that they should definitely not be treated as two-level systems - even though their binding energies are closer to those of the more molecule-like Frenkel excitons. Bosonic creation and annihilation operators $\hat{b}^{\dagger}$ and $\hat{b}$ generate a new electron-hole pair going one step up the bosonic ladder, as shown in the figure within a rather simplified picture where exciton interactions and formation of bi-excitons [84, 85] are disregarded for simplicity [121]. It is therefore important to depart from the picture of excitons being analogous to a hydrogen atom, and distinguish between physical entities and quasiparticles.

One could argue that the fundamental issue regarding the nature of the coupled components can be bypassed by the transformation of $N$ two-level systems to a bosonic super-oscillator, as discussed in Subsection ЩA and shown in the bottom schematics of Figure 2. This is valid, provided that the system is in the linear response regime (low number of excitations so that saturation effects are irrelevant, which is implicitly assumed throughout this manuscript). But apart from this, several practical problems arise in such calculations. As discussed above, the first one appears in the form of the definition of the cavity: the dipole moment of TMDs is strictly restricted in the two-dimensional material layer, and aligned in plane. Evaluating the energy density everywhere in space around a plasmonic NP on top of such a TMD [86] is thus really not relevant. Furthermore, in estimations based on $N / V$ one typically assumes that the coupling strength is that corresponding to an emitter located at the position of the maximum field, which is definitely inaccurate for a large collection of excitations distributed over a large area and inside the inhomoge- 


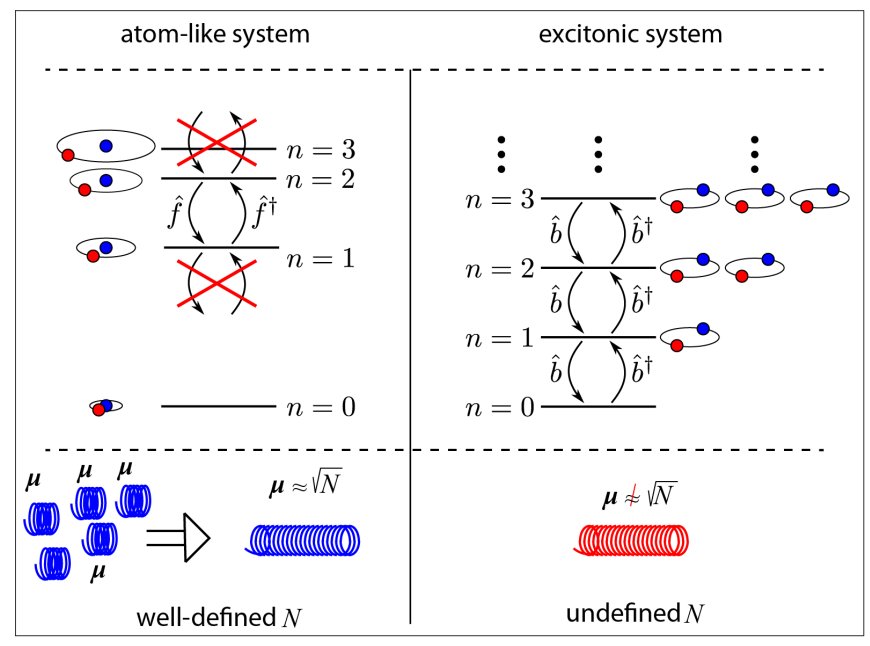

FIG. 2: Differences between the types of emitters employed in strong-coupling schemes in nanophotonics. Upper panels: On the left, the energy ladder of a two-level system; the presence of $N$ emitters (e.g. atoms) in the cavity means exactly $N$ such two-level systems (see schematics of Figure1) interacting with the cavity field (and possible with each other, so-called Dicke superradiance). On the right, the energy ladder of a bosonic system (e.g. excitons in TMDs). Adding one exciton means creating one additional boson and going one step up the ladder, with all excitons sharing the same quantum numbers and contributing to the collective excitonic state that interacts with the cavity. Interactions between excitons and formation of more complex quasiparticles like bi-excitons or trions have been disregarded for the sake of simplicity. Lower panels: On the left, $N$ independent two-level systems with dipole moment $\boldsymbol{\mu}$ combine in the low-excitation limit to form a super-oscillator whose dipole moment is proportional to $\sqrt{N}$. On the right, the strong collective dipole moment of an excitonic system is not the result of the combination of $N$ specific dipoles.

neous near field of a plasmonic antenna of any shape. The dipole moment in such a calculation can also be ambiguous, and it is tempting to use equation (1) as its indirect measure [87]. One could raise the question about the nature of the matter excitations the cavity couples to: are they individual, site-localised excitons, or an excitonic state extended in space and involving a number of bound electron-hole pairs in the TMD sheet? As already pointed out by others [87], only the latter can account for the strong dipole moment achieved in such systems. While this seems again to be the case of a super-oscillator, similar to that of $N$ two-level atoms treated by the Tavis-Cummings Hamiltonian, the key difference is that excitons are delocalised or, at best, only localised over many unit-cells in the TMD and do not correspond to a specific number $N$, as shown in the bottom schematics of Figure 2, whereas the dipole moment of the molecular super-oscillator is indeed proportional to $\sqrt{N}$. This super-oscillator response was the charm of $J$-aggregates in the first place, with dipole moments aligning to create the stronger effective dipole responsi- ble for the anticrossing in the spectra. But then it is clear that a calculation of a number of excitons uniquely on the basis of the dipole moment of a single exciton is misleading and highly questionable. One should thus be particularly careful not to mix extended hybrid states with transitions in distinguishable entities.

Such ambiguities have led to estimations of very different numbers of excitons for very similar systems, most strikingly in the case of metallic nanorods on top of tungsten-based TMDs, where in [27] a number of about 5 excitons was estimated, while in [26] the corresponding value was of the order of a few thousands. Despite the obvious inconsistency, the apparent appeal of such calculations persists, and they are now adopted even in other contexts where no emitters are present, to evaluate for instance the dipole moment of individual plasmons in metallic nanodisc arrays, where each disc is essentially treated as the equivalent of a two-level system [88], using the standard terminology of the Rabi problem. The problems with this approach are that a) not every hybridisation gap can be called a Rabi splitting, neither can every excitation (in this case the plasmon) be assimilated to an idealised (two-level) system with a transition. This might seem like a matter of terminology, but it is exactly the kind of approach that can lead to the unfounded extensions of concepts discussed in this Review. b) In any case, the disc array supports a collective plasmonic mode [89] and conclusions about individual dipole moments cannot be judiciously drawn. While estimating a dubious exciton number does not provide any particularly useful information in this context of strong coupling (at least in the majority of scenarios), if one insists, there are still more accurate ways than through $N / V$ to do this. An example of a more appropriate approach can by found in [26] where, starting from the first equality in equation (10), the authors evaluated a local coupling, at position $\mathbf{r}_{i}$, through $\boldsymbol{\mu}\left(\mathbf{r}_{i}\right) \cdot \mathbf{E}\left(\mathbf{r}_{i}\right)$ and summing over all local $g$ values:

$$
g=\sqrt{\sum_{i} g\left(\mathbf{r}_{i}\right)^{2}} .
$$

Since the EM field decays exponentially away from the plasmonic nanostructure, the sum in equation (5) will eventually converge to some value, which in [26] agreed rather well with a rough estimate based on the single exciton radius and the area below the metallic nanorod. Such a calculation could be useful in describing the system in terms of the equivalent image of individual excitons that would produce the same response - or, in other words, an effective exciton. Nevertheless, even this approach contains the somehow vague notation $i$ : a space discretisation immediately reduces the problem to the traditional picture of $N$ two-level systems, and if all their dipole moments are assumed equal, aligned, and experiencing the same field, one retrieves equation (11). But to use this for a very rough estimate, one can only base it on the assumption that each exciton occupies a specific space in 
the TMD sheet (typically defined by its Bohr radius), an assumption in strong conflict with the collective character of the excitation. A more strict calculation should include an integral of the coupling strength over the entire TMD sheet area $\mathcal{S}$ [90]

$$
\int_{\mathcal{S}} \mathrm{d}^{2} r|g(\mathbf{r})|^{2}
$$

as we briefly show with a simple toy model in the next Subsection. But the main message of this part is that one should not try to extend the analogies between excitonic states and two-level systems beyond certain limits.

\section{Size of excitons: what is the coupling strength?}

In the previous Subsection we pointed out that excitons, for example in atomically-thin TMDs, should not be confused with atoms, as their number is not an inherent property of the system itself (i.e., the Hamiltonian), but a consequence of the illumination - and it should be clear that all the treatments discussed here only apply in the low excitation, linear-response case anyway. A second misunderstanding that can easily develop from the exciton-atom analogy is that, despite the illustrative picture of a single exciton as a bound state of orbiting electron and hole (Figure 2), the excitonic state in a TMD coupled to a plasmonic resonator is in fact not localised at the excitonic Bohr radius - one of the reasons being exactly the fact that the plasmon-exciton interaction gives rise to new, hybrid states, which are evidently dissimilar to the initial bare states. This implies that the effective plasmon-exciton coupling cannot be estimated via the maximal field enhancement of the plasmonic structure, a treatment based on the picture of excitons as small movable atom-like objects that accumulate in the plasmonic hotspot and all couple according to the maximally attainable electric field. This treatment is inaccurate, at least within the regime where the optical response of the TMD is approximately linear, i.e. for low to moderate optical excitation intensities. To illustrate this, we introduce a very simple model, where we bypass the mode volume issue by assuming a lossless closed cavity. Although our description is highly idealised and in principle well-known [91, 92], it is capable of explicitly showing how extended excitonic states emerge quite naturally even from a model based on non-interacting and localised excitons. While there are analogies with the Dicke [93] or Tavis-Cummings [14] models for two-level emitters, these are not identical cases 122 .

We consider the interaction of an optical mode described by ladder operators $\hat{a}$ and $\hat{a}^{\dagger}$ with localised exciton states that can be created and annihilated at any point $\mathbf{r}$ within the TMD via the operators $\hat{b}_{\mathbf{r}}^{\dagger}$ and $\hat{b}_{\mathbf{r}}$, respectively. The state of an exciton centred at $\mathbf{r}$ is denoted as $|\mathbf{r}\rangle$, while the single-photon state of the resonator as $|a\rangle$. We further assume that $a$ ) both the closed cavity and the excitonic layer support normalisable modes without damping, and b) there is no interaction between the excitons. The latter is just a reformulation of our assumption of moderate pumping intensities, whereas the former is a somewhat crude assumption for the sake of simplicity. In closed cavities losses can be introduced via Lindblad operators 95]. In open cavities, and/or in the presence of dissipation, things become even more complicated, but steps towards an accurate description have been taken recently [96]. A more precise analysis where interactions between excitons are taken into account is feasible [97], but it exceeds the purely illustrative purposes of this section.

Within the rotating wave approximation, the excitoncavity Hamiltonian is

$$
\mathcal{H}=\hbar \omega_{0} \hat{a}^{\dagger} \hat{a}+\int_{\mathcal{S}} \mathrm{d}^{2} r\left\{\hbar \Omega \hat{b}_{\mathbf{r}}^{\dagger} \hat{b}_{\mathbf{r}}+\left[g(\mathbf{r}) \hat{a}^{\dagger} \hat{b}_{\mathbf{r}}+\text { h.c. }\right]\right\},
$$

where $\omega_{0}$ is the angular frequency of the cavity mode, $\Omega$ the angular frequency of the degenerate excitons, and h.c. denotes the Hermitian conjugate of the expression in square brackets. An exciton at $\mathbf{r}$ couples to the cavity mode via a matrix element $g(\mathbf{r})$, whose exact form is of no concern in this context. In the case of distinguishable emitters it is usually sufficient to employ the electric dipole approximation as $g(\mathbf{r})=\boldsymbol{\mu} \cdot \mathbf{E}(\mathbf{r})$, where $\boldsymbol{\mu}$ denotes the exciton dipole moment and $\mathbf{E}(\mathbf{r})$ is the electric field of the optical mode. In order to diagonalise this Hamiltonian, we introduce a family $h_{\alpha}(\mathbf{r})$ of functions that are orthogonal to $g(\mathbf{r})$ and such that the set $\left\{g(\mathbf{r}), h_{\mathbb{R}}(\mathbf{r})\right\}$ constitutes a basis for the space $L^{2}(\mathcal{S})$. This should always be possible if the cavity mode is square-integrable in the sheet.

We now transform the excitonic system into this new basis $|g\rangle=N_{g} \int_{\mathcal{S}} \mathrm{d}^{2} r \quad g^{*}(\mathbf{r})|\mathbf{r}\rangle$, and $|\alpha\rangle=$ $N_{\alpha} h_{\alpha}^{*}(\mathbf{r})|\mathbf{r}\rangle$, where the normalisation constants are $N_{g}=$ $\left[\int_{\mathcal{S}} \mathrm{d}^{2} r|g(\mathbf{r})|^{2}\right]^{-1 / 2}, N_{\alpha}=\left[\int_{\mathcal{S}} \mathrm{d}^{2} r\left|h_{\alpha}(\mathbf{r})\right|^{2}\right]^{-1 / 2}$. By applying the Hamiltonian to the orthogonalised states $|\alpha\rangle$, and using $\hat{a}|\mathbf{r}\rangle=0$ and $\hat{b}_{\mathbf{r}}\left|\mathbf{r}^{\prime}\right\rangle=\delta\left(\mathbf{r}-\mathbf{r}^{\prime}\right)|0\rangle$, we find $\mathcal{H}|\alpha\rangle=\hbar \Omega|\alpha\rangle$. Obviously these states do not couple to the resonator mode, because their spatial envelope was constructed to be orthogonal to the coupling distribution $g(\mathbf{r})$, and could be called cavity-dark exciton states. As a result, the light-matter coupling problem reduces to the two remaining states $|a\rangle$ and $|g\rangle$. Applying the Hamiltonian we obtain $\mathcal{H}|a\rangle=\hbar \omega_{0}|a\rangle+N_{g}^{-1}|g\rangle$ and $\mathcal{H}|g\rangle=\hbar \Omega|g\rangle+N_{g}^{-1}|a\rangle$. This means that the eigenstates can be written as $c_{a}|a\rangle+c_{g}|g\rangle$, where the coefficients are given by the eigenvalue problem

$$
\hbar\left(\begin{array}{cc}
\omega_{0} & \kappa \\
\kappa & \Omega
\end{array}\right)\left(\begin{array}{l}
c_{a} \\
c_{g}
\end{array}\right)=\mathcal{E}\left(\begin{array}{c}
c_{a} \\
c_{g}
\end{array}\right),
$$

with the real-valued $\kappa=\left(\hbar N_{g}\right)^{-1}$. The eigenenergies are

$$
\mathcal{E}_{ \pm}=\hbar \frac{\omega_{0}+\Omega}{2} \pm \hbar \frac{\sqrt{\left(\omega_{0}-\Omega\right)^{2}+\kappa^{2}}}{2}=\hbar \Omega \pm \frac{\hbar \Omega_{\mathrm{B}}}{2}
$$

where the second equality holds on resonance, with coef- 
ficients $c_{a}= \pm c_{g}=\sqrt{1 / 2}$ and beat frequency

$$
\hbar \Omega_{\mathrm{B}}=\sqrt{\int_{\mathcal{S}} \mathrm{d}^{2} r|g(\mathbf{r})|^{2}},
$$

which contains the expression suggested in equation (6). Similar analyses were presented recently in [65] and [96]. The corresponding problem for $N$ two-level quantum emitters in a plasmonic cavity (where the cavity was indeed such, consisting of metal films), was solved in [98], while a computationally efficient model that allows to describe a large number of two-level emitters in a microcavity was introduced in 94. While all these approaches might share some common points, the key difference is the origin and meaning of the collective dipole moments involved in the coupling.

The main message from the present analysis is that the eigenstates of the Hamiltonian are hybrid states that have both plasmonic and excitonic character, and the excitonic part itself is a hybridisation of many excitons delocalised throughout the entire TMD sheet. This is not exactly surprising, as it corresponds to two harmonic oscillators coupled to each other, where the eigenstates emerge as the hybridised states, and the linearity of both subsystems guarantees that these new states are excited quantum-by-quantum. There is not much sense in counting the number $N$ of excitons, because their number is strictly linked to the photon number - and thus dependent on the illumination intensity — via the hybrid eigenstate.

\section{DISCUSSION AND CONCLUSIONS}

The preceding analysis does not imply that strongcoupling nanophotonics went astray in its entirety and should be completely revisited: on the contrary, this is a very fruitful area of research, where many important results have been derived and exciting applications suggested [99]. For instance, quantum statistical phenomena such as Bose-Einstein condensation and polariton lasing do not necessitate operation at the single-emitter or single-photon limit, on the contrary, they require a large number of excitations 100 106. Similarly, nonlinearities and entanglement [107] can be explored without the need for single excitons. In any case, if one wants to maximise the coupling, classical physics already provides the guidelines: strong modes (EM fields) with significant overlap are the safest way to increase the interaction in any system effectively described as a pair of coupled harmonic oscillators. Furthermore, for quantum applications, what one really needs in practice is single photons [108, 109], regardless of the way they where generated, and the questionable single excitons in organic molecules or TMDs are not, by default, a necessary condition to achieve this goal. Consequently, it makes much more sense to focus for example on whether antibunching is observed, and try to measure quantities such as the photon emission rate and the second-order correlation function, as was done very recently for other plasmon-emitter systems [110 112].

Another exciting direction that is drawing significant attention recently is polaritonic chemistry [113], which focuses on the possibility to manipulate chemical reactions and structures through the formation of polaritons. Initiated by the seminal work of the Ebbesen group [114], this area is now shifting from the usual dipole approximations to consider all internal degrees of freedom (electronic, vibrational, nuclear) of the molecules [115], calling thus for a rigorous theoretical description, possibly within timedependent density-functional theory [92, 116, 117], where many of the assumptions criticised here are absent by construction.

Throughout this manuscript, whenever discussing a treatment that we considered erroneous, we always tried to also mention references containing what we consider as the corresponding correct description, or at least a right step towards that. At first, it might appear that we claim that theorists are usually correct while experimentalists are prone to errors. This does definitely not reflect our view of the field: papers that were criticised here for some reason, still contain otherwise excellent work. For example, a paper with whose mode-volume estimation we disagreed [61] is nevertheless a pioneering case of designing plasmon-exciton hybrids operating at room temperature, and also discusses thoroughly the criteria for reaching strong coupling [118]. Our feeling is that questionable extension of ideas has occurred mostly as a result of pressure to differentiate new manuscripts by adding an extra flavour. In the absence of a robust theoretical description, approximations have therefore been made based on the theories already available. This is of course acceptable, as long as no attempt to interpret such estimates as exact quantified results is made.

In summary, we have discussed situations where concepts from cQED have been used to describe strong coupling in nanophotonics - and plasmonics in particular - without this use being fully justified. We showed that while basing qualitative discussions on the factor $N / V$ can be a good starting point, as long as this factor is interpreted as an effective density of emitters, in most prevalent architectures in current literature neither $V$ nor $N$ are well defined, and quantitative conclusions uniquely based on these quantities should be avoided, especially when excitons in TMDs are involved. Instead, one should go one step backwards, deduce experimentally relevant information directly from the coupling of the standard dipole approximation, and then measure if the system exhibits the desired quantum response. We hope that the discussion presented here, even if it appears overly critical at points, will help the nanophotonics community to set its future efforts on a more solid foundation. 


\section{Acknowledgments}

N. A. M. is a VILLUM Investigator supported by VILLUM FONDEN (grant No. 16498). S. I. B. acknowledges the European Research Council (grant 341054, PLAQNAP). The Center for Nano Optics is financially supported by the University of Southern Denmark (SDU 2020 funding). The Center for Nanostructured Graphene is sponsored by the Danish National Research Foundation (Project No. DNRF103). F. T. and C. W. ac- knowledge funding from MULTIPLY fellowships under the Marie Skłodowska-Curie COFUND Action (grant agreement No. 713694). K. B. acknowledges funding from the Deutsche Forschungsgemeinschaft (DFGProjektnummer 182087777-SFB 951 "HIOS"). We are grateful to L. Lavazza for stimulating the work and we thank T. Shegai and J. Aizpurua, and the anonymous referees for highly constructive feedback on the manuscript and inspiring discussions.
[1] J. D. Joannopoulos, S. G. Johnson, J. W. Winn, and R. D. Meade, Photonic Crystals: Molding the Flow of Light (2nd Edition) (Princeton University Press, Princeton, 2008).

[2] M. Segev, Y. Silderberg, and D. N. Christodoulides, Nat. Photon. 7, 197 (2013).

[3] S. Zhang, D. A. Genov, Y. Wang, M. Liu, and X. Zhang, Phys. Rev. Lett. 101, 047401 (2008).

[4] L. Mandel and E. Wolf, Quantum Coherence and Quantum Optics (Cambridge University Press, Cambridge, 1995).

[5] S. Haroche and D. Kleppner, Phys. Today 42(1), 24 (1989).

[6] C. Weisbuch, M. Nishioka, A. Ishikawa, and Y. Arakawa, Phys. Rev. Lett. 69, 3314 (1992).

[7] T. Yoshie, A. Scherer, J. Hendrickson, G. Khitrova, H. M. Gibbs, G. Rupper, C. Ell, O. B. Shchekin, and D. G. Deppe, Nature 432, 200 (2004).

[8] J. Bellessa, C. Bonnand, J. C. Plenet, and J. Mugnier, Phys. Rev. Lett. 93, 036404 (2004).

[9] X. Liu, T. Galfsky, Z. Sun, F. Xia, E.-C. Lin, Y.-H. Lee, S. Kéna-Cohen, and V. M. Menon, Nat. Photon. 9, 30 (2015).

[10] W. Liu, Y. Wang, C. H. Naylor, B. Lee, B. Zheng, G. Liu, A. T. C. Johnson, A. Pan, and R. Agarwal, ACS Photonics 5, 192 (2018).

[11] O. Astafiev, A. M. Zagoskin, A. A. Abdumalikov Jr., Y. A. Pashkin, T. Yamamoto, K. Inomata, Y. Nakamura, and J. S. Tsai, Science 327, 840 (2010).

[12] D. Sanvitto and S. Kéna-Cohen, Nat. Mater. 15, 1061 (2016).

[13] P. Törmä and W. L. Barnes, Rep. Prog. Phys. 78, 013901 (2015).

[14] M. Tavis and F. W. Cummings, Phys. Rev. 170, 379 (1968).

[15] M. O. Scully and M. S. Zubairy, Quantum Optics (Cambridge University Press, Cambridge, 1997).

[16] L. Shi, T. K. Hakala, H. T. Rekola, J.-P. Martikainen, R. J. Moerland and P. Törmä Phys. Rev. Lett. 112, 153002 (2014).

[17] J. Dintinger, S. Klein, F. Bustos, W. L. Barnes, and T. W. Ebbesen, Phys. Rev. B 71, 035424 (2005).

[18] Y. Sugawara, T. A. Kelf, J. J. Baumberg, M. E. Abdelsalam, and P. N. Bartlett, Phys. Rev. Lett. 97, 266808 (2006).

[19] R. Chikkaraddy, B. de Nijs, F. Benz, S. J. Barrow, O. A. Scherman, E. Rosta, A. Demetriadou, P. Fox, O. Hess, and J. J. Baumberg, Nature 535, 127 (2016).
[20] D. G. Baranov, M. Wersäll, J. Cuadra, T. J. Antosiewicz, and T. Shegai, ACS Photonics 5, 24 (2018).

[21] T. Schwartz, J. A. Hutchison, C. Genet C and T. W. Ebbesen, Phys. Rev. Lett. 106, 196405 (2011).

[22] A. Cacciola, O. Di Stefano, R. Stassi, R. Saija, and S. Savasta, ACS Nano 8, 11483 (2014).

[23] F. Todisco, M. De Giorgi, M. Esposito, L. De Marco, A. Zizzari, M. Bianco, L. Dominici, D. Ballarini, V. Arima, G. Gigli, and D. Sanvitto, ACS Photonics 5, 143 (2018).

[24] J. T. Hugall, A. Singh, and N. F. van Hulst, ACS Photonics 5, 43 (2018).

[25] J. B. Khurgin, Nat. Nanotechnol. 10, 2 (2015).

[26] D. Zheng, S. Zhang, Q. Deng, M. Kang, P. Nordlander, and H. Xu, Nano Lett. 17, 3809 (2017).

[27] J. Wen, H. Wang, W. Wang, Z. Deng, C. Zhuang, Y. Zhang, F. Liu, J. She, J. Chen, H. Chen, S. Deng, and N. Xu, Nano Lett. 17, 4689 (2017).

[28] X. Han, K. Wang, X. Xing, M. Wang, and P. Lu, ACS Photonics 5, 3970 (2018).

[29] J. Cuadra, D. G. Baranov, M. Wersäll, R. Verre, T. J. Antosiewicz, and T. Shegai, Nano Lett. 18, 1777 (2018).

[30] C. Tserkezis, M. Wubs, and N. A. Mortensen, ACS Photonics 5, 133 (2018).

[31] G. Khitrova, H. M. Gibbs, M. Kira, S. W. Koch, and A. Scherer, Nat. Phys. 2, 18 (2006).

[32] H. Bahsoun, T. Chervy, A. Thomas, K. Börjesson, M. Hertzog, J. George, E. Devaux, C. Genet, J. A. Hutchison and T. W. Ebbesen, ACS Photonics 5, 225 (2018).

[33] Y. Kaluzny, P. Goy, M. Gross, J. M. Raimond, and S. Haroche, Phys. Rev. Lett. 51, 1175 (1983).

[34] W. Gao, X. Li, M. Bamba, and J. Kono, Nat. Photon. 12, 362 (2018).

[35] J. D. Jackson, Classical Electrodynamics, 3rd edn (Wiley, Hoboken, 1999).

[36] T. K. Hakala, J. J. Toppari, A. Kuzyk, M. Pettersson, H. Tikkanen, H. Kunttu, and P. Törmä, Phys. Rev. Lett. 103, 053602 (2009).

[37] D. F. Walls and G. J. Millburn, Quantum Optics (Springer, Berlin, 1994).

[38] G. S. Agarwal Quantum Optics (Cambridge University Press, Cambridge, 2012).

[39] Y. Kurman, N. Rivera, T. Christensen, S. Tsesses, M. Orenstein, M. Soljačić, J. D. Joannopoulos, and I. Kaminer, Nat. Photon. 12, 423 (2018).

[40] A. Cuartero-González and A. I. Fernández-Domínguez, ACS Photonics 5, 3415 (2018).

[41] G. Rempe, H. Walther, and N. Klein, Phys. Rev. Lett. 58, 353 (1987). 
[42] R. J. Thompson, G. Rempe, and H. J. Kimble, Phys. Rev. Lett. 68, 1132 (1992).

[43] A. B. Yankovich, B. Munkhbat, D. G. Baranov, J. Cuadra, E. Olsén, H. Lourenço-Martins, L. H. G. Tizei, M. Kociak, E. Olsson, and T. Shegai, Nano Lett. 19, 8171 (2019).

[44] J.-M. Gérard and B. Gayral, J. Lightwave Technol. 17, 2089 (1999).

[45] L. Novotny and B. Hecht, Principles of Nano-Optics (Cambridge University Press, Cambridge, 2006).

[46] R. Ruppin, Phys. Lett. A 299, 309 (2002).

[47] S. A. Maier, Opt. Express 14, 1957 (2006).

[48] C. Sauvan, J. P. Hugonin, I. S. Maksymov, and P. Lalanne, Phys. Rev. Lett. 110, 237401 (2013).

[49] P. T. Kristensen, C. Van Vlack, and S. Hughes, Opt. Lett. 37, 1649 (2012).

[50] M. Kamandar Dezfouli and S. Hughes, Phys. Rev. B 97, 115302 (2018).

[51] M. Kamandar Dezfouli, C. Tserkezis, N. A. Mortensen and S. Hughes, Optica 4, 1503 (2017).

[52] P. T. Leung, S. Y. Liu and K. Young, Phys. Rev. A 49, 3982 (1994).

[53] P. T. Kristensen, R.-C. Ge, and S. Hughes, Phys. Rev. A 92, 053810 (2015).

[54] W. Yan, R. Faggiani and P. Lalanne, Phys. Rev. B 97, 205422 (2018).

[55] P. Lalanne, W. Yan, A. Gras, C. Sauvan, J,-P. Hugonin, M. Besbes, G. Demésy, M. D. Truong, B. Gralak, F. Zolla, A. Nicolet, F. Binkowski, L. Zschiedrich, S. Burger, J. Zimmerling, R. Remis, P. Urbach, H. T. Liu and T. Weiss, J. Opt. Soc. Am. A 36, 686 (2019).

[56] B. J. Dalton, S. M. Barnett and B. M. Garraway, Phys. Rev. A 64, 053813 (2001).

[57] Y. Yang, D. Zhu, W. Yan, A. Agarwal, M. Zheng, J. D. Joannopoulos, P. Lalann, T. Christensen, K. K. Berggren and M. Soljačić, Nature 576, 248 (2019).

[58] P. T. Kristensen and S. Hughes, ACS Photon. 1, 2 (2014).

[59] P. Lalanne, W. Yan, K. Vynck, C. Sauvan, and J.-P. Hugonin, Laser Photon. Rev. 12, 1700113 (2018).

[60] P. T. Kristensen, K. Herrmann, F. Intravaia and K. Busch, Adv. Opt. Photon., DOI: 10.1364/AOP.377940 (2020).

[61] G. Zengin, M. Wersäll, S. Nilsson, T. J. Antosiewicz, M. Käll, and T. Shegai, Phys. Rev. Lett. 114, 157401 (2015).

[62] A. F. Koenderink, Opt. Lett. 35, 4208 (2010).

[63] T. V. Shahbazyan, Phys. Rev. B 98, 115401 (2018).

[64] A. Delga, J. Feist, J. Bravo-Abad, and F. J. GarcíaVidal, Phys. Rev. Lett. 112, 253601 (2014).

[65] T. V. Shahbazyan, Nano Lett. 19, 3273 (2019).

[66] M. Ramezani, G. Lozano, M. A. Verschuuren, and J. Gómez-Rivas, Phys Rev. B 94, 125406 (2016).

[67] E.-M. Roller, C. Argyropoulos, A. Högele, T. Liedl, and M. Pilo-Pais, Nano Lett. 16, 5962 (2016).

[68] E. M. Purcell, Phys. Rev. 69, 681 (1949).

[69] P. Anger, P. Bharadwaj, and L. Novotny, Phys. Rev. Lett. 96, 113002 (2006).

[70] R. Ruppin, J. Chem. Phys. 76, 1681 (1982).

[71] N. Kongsuwan, A. Demetriadou, R. Chikkaraddy, F. Benz, V. A. Turek, U. F. Keyser, J. J. Baumberg and O. Hess, ACS Photonics 5, 186 (2018).

[72] I. M. Palstra, H. M. Doeleman, and A. F. Koenderink, Nanophotonics, DOI: 10.1515/nanoph-2019-
0062 (2019).

[73] R. F. Oulton, G. Bartal, D. F. P. Pile, and X. Zhang, New J. Phys. 10, 105108 (2008).

[74] K. Santhosh, O. Bitton, L. Chuntonov and G. Haran Nat. Commun. 7, 11823 (2016).

[75] G. Kewes, F. Binkowski, S. Burger, L. Zschiedrich and O. Benson, ACS Photon. 5, 4089 (2018).

[76] F. Stete, P. Schoßau, M. Bargheer, and W. Koopman, J. Phys. Chem. C 122, 17976 (2018).

[77] I. Pockrand, A. Brillante, and D. Möbius, J. Chem. Phys. 77, 6289 (1982).

[78] G. Wang, A. Chernikov, M. M. Glazov, T. F. Heinz , X. Marie, T. Amand and B. Urbaszek Rev. Mod. Phys. 90, 021001 (2018).

[79] J. J. Hopfield, Phys. Rev. 112, 1555 (1958).

[80] M. Combescot and O. Betbeder-Matibet, EPL 59, 579 (2002).

[81] B. Laikhtman, J. Phys.: Condens. Matter 19, 295214 (2007).

[82] H. Yu, X. Cui, X. Xu and W. Yao, Ntl. Sci. Rev. 2, 57 (2015).

[83] P. Cudazzo, L. Sponza, C. Giorgetti, L. Reining, F. Sottile and M. Gatti, Phys. Rev. Lett. 116, 066803 (2016).

[84] K. F. Mak and J. Shan, Nat. Photon. 10216 (2016).

[85] Y.0M. He, O. Iff, N. Lundt, V. Baumann, M. Davanco, K. Srinivasan, S. Höfling and C. Schneider, Nat. Commun. 713409 (2016).

[86] S. Hou, L. Y. M. Tobing, X. Wang, Z. Xie, J. Yu, J. Zhou, D. Zhang, C. Dang, P. Coquet, B. K. Tay, M. D. Birowosuto, E. H. T. Teo and H. Wang, Adv. Opt. Mater. 7, 1900857 (2019).

[87] M. Stührenberg, B. Munkhbat, D. G. Baranov, J. Cuadra, A. B. Yankovich, T. J. Antosiewicz, E. Olsson, and T. Shegai, Nano Lett. 18, 5938 (2018).

[88] A. Bisht, J. Cuadra, M. Wersäll, A. Canales, T. J. Antosiewicz, and T. Shegai, Nano Lett. 19, 189 (2019).

[89] C. Tserkezis, G. Gantzounis, and N. Stefanou, J. Phys.: Condens. Matter 20, 075232 (2008).

[90] A. E. Schlather, N. Large, A. S. Urban, P. Nordlander and N. J. Halas, Nano Lett. 13, 3281 (2013).

[91] S. Dhara, C. Chakraborty, K. M. Goodfellow, L. Qiu, T. A. O'Loughlin, G. W. Wicks, S. Bhattacharjee, and A. N. Vamivakas, Nat. Phys. 14, 130 (2018).

[92] J. Flick, N. Rivera, and P. Narang, Nanophotonics 7, 1479 (2018).

[93] R. H. Dicke, Phys. Rev. 93, 99 (1954).

[94] R. Trivedi, M. Radulaski, K. A. Fischer, S. Fan and J. Vučković, Phys. Rev. Lett. 122, 243602 (2019).

[95] T. Neuman and J. Aizpurua, Optica 5, 1247 (2018).

[96] S. Franke, S. Hughes, M. Kamandar Dezfouli, P. T. Kristensen, K. Busch, A. Knorr, and M. Richter, Phys. Rev. Lett. 122, 213901 (2019).

[97] V. M. Agranovich, M. Litinskaia, and D. G. Lidzey, Phys. Rev. B 67, 085311 (2003).

[98] A. González-Tudela, P. A. Huidobro, L. Martín-Moreno, C. Tejedor, and F. J. García-Vidal, Phys. Rev. Lett. 110, 126801 (2013).

[99] A. I. Fernández-Domínguez, S. I. Bozhevolnyi, and N. A. Mortensen, ACS Photonics 5, 3447 (2018).

[100] A. Amo, D. Sanvitto, F. P. Laussy, D. Ballarini, E. del Valle, M. D. Martin, A. Lemaître, J. Bloch, D. N. Krizhanovskii, M. S. Skolnick, C. Tejedor, and L. Viña, Nature 457, 291 (2009).

[101] K. S. Daskalakis, S. A. Maier, R. Murray, and S. Kéna- 
Cohen, Nat. Mater. 13, 271 (2014).

[102] T. K. Hakala, A. J. Moilanen, A. I. Väkeväinen, R. Guo, J.-P. Martikainen, K. S. Daskalakis, H. T. Rekola, A. Julku, and P. Törmä, Nat. Phys. 14, 739 (2018).

[103] S. Kéna-Cohen and S. R. Forrest, Nat. Photon. 4, 371 (2010).

[104] D. Ballarini, M. De Giorgi, E. Cancellieri, R. Houdré, E. Giacobino, R. Cingolani, A. Bramati, G. Gigli, and D. Sanvitto, Nat. Commun. 4, 1178 (2013).

[105] A. I. Väkeväinen, A. J. Moilanen, M. Nečada, T. K. Hakala, K. S. Daskalakis and P. Törmä, Nat. Com. 11, 3139 (2020). 1905.07609

[106] M. Ramezani, A. Halpin, A. I. Fernández-Domínguez, J. Feist, S. R.-K. Rordriguez, F. J. García-Vidal, and J. Gómez-Rivas, Optica 4, 31 (2017).

[107] J. M. Raimond, M. Brune, and S. Haroche, Rev. Mod. Phys. 73, 565 (2001).

[108] P. Lodahl, S. Mahmoodian and S. Stobbe, Rev. Mod. Phys. 87, 347 (2015).

[109] M. Pelton, Nat. Photon. 9, 427 (2015).

[110] R. Sáez-Blázquez, J. Feist, A. I. Fernández-Domínguez, and F. J. García-Vidal, Optica 4, 1363 (2017).

[111] A. Singh, P. M. de Roque, G. Calbris, J. T. Hugall, and N. F. van Hulst, Nano Lett. 18, 2538 (2018).

[112] O. S. Ojambati, R. Chikkaraddy, W. D. Deacon, M. Horton, D. Kos, V. A. Turek, U. F. Keyser, and J. J. Baumberg, Nat. Commun. 10, 1049 (2019).

[113] J. Feist, J. Galego, and F. J. García-Vidal, ACS Photonics 5, 205 (2018).

[114] J. A. Hutchison, T. Schwartz, C. Genet, E. Devaux, and T. W. Ebbesen, Angew. Chem. 51, 1592 (2012).
[115] J. Galego, F. J. García-Vidal, and J. Feist, Phys. Rev. X 5, 041022 (2015).

[116] T. Neuman, R. Esteban, D. Casanova, F. J. GarcíaVidal, and J. Aizpurua, Nano Lett. 18, 2358 (2018).

[117] T. P. Rossi, T. Shegai, P. Erhart, and T. J. Antosiewicz, Nat. Com. 10, 3336 (2019).

[118] G. Zengin, T. Gschneidtner, R. Verre, L. Shao, T. J. Antosiewicz, K. Moth-Poulsen, M. Käll, and T. Shegai, J. Phys. Chem. C 120, 20588 (2016).

[119] Note that in these references use of equation (1) was fully justified.

[120] It should be noted here that both classical and semiclassical descriptions have been obtained within certain approximations, and deviations from the $\sqrt{N / V}$ dependence might be observed for small numbers of emitters in small volumes - when a macroscopic density is not well defined. Nevertheless, this dependence has been observed so frequently in experiments, that any microscopic model is expected to lead to a similar response.

[121] Here it is important to distinguish excitons in, say, quantum dots - where any electron-hole pair is (or, rather, becomes) an exciton - and in TMDs, where there is a crucial difference between an excited electronhole pair (single-particle picture, no interactions) and an exciton (many electron picture, screened interaction).

[122] And even if they were, a direct transition from the Tavis-Cummings to the Jaynes-Cummings description is not always straightforward [94]. 